\title{
Joint Applied Optics and Chinese Optics Letters feature introduction: digital holography and three-dimensional imaging
}

\author{
Ting-Chung Poon \\ The Bradley Department of Electrical and Computer Engineering, Virginia Tech, \\ Blacksburg, Virginia 24061, USA (tcpoon@vt.edu) \\ Received 23 November 2011; accepted 23 November 2011; \\ posted 23 November 2011 (Doc. ID 158362); published 1 December 2011
}

\begin{abstract}
This feature issue serves as a pilot issue promoting the joint issue of Applied Optics and Chinese Optics Letters. It focuses upon topics of current relevance to the community working in the area of digital holography and 3-D imaging. (C) 2011 Optical Society of America OCIS codes: $\quad 010.0280,020.1335$.
\end{abstract}

This feature issue is the fifth installment on digital holography since its inception four years ago. The past four issues have been published after the conclusion of each Topical Meeting "Digital Holography and 3-D imaging (DH)." However, this feature issue includes a new key feature-the Joint Applied Optics and Chinese Optics Letters Feature Issue.

The DH Topical Meeting is the world's premier forum for disseminating the science and technology geared toward digital holography and 3-D information processing. Since the meeting's inception in 2007, it has steadily and healthily grown to 130 presentations this year, held in Tokyo, Japan, May 2011.

The Topical Meeting has been providing a forum for disseminating the science and technology of holographic interferometry for deformation or contour measurement, new technologies for phase unwrapping, 3-D optical remote sensing, 3-D holographic microscopy, 3-D optical image processing, 3-D display, and digital holography for life science or nanophotonics applications. Again, this joint feature issue includes a representative selection of topics that have been presented at the DH Topical Meeting in Tokyo.

One of the key elements of the present joint feature issue is that the issue serves as a pilot issue promoting the joint issue of Applied Optics (AO)

0003-6935/11/340DH1-02 $\$ 15.00 / 0$

(C) 2011 Optical Society of America and Chinese Optics Letters (COL). This joint feature issue is a result of face-to-face discussion with the Executive Editor-in-Chief of Chinese Optics Letters Professor Changhe Zhou (when Poon had the opportunity to visit Professor Zhou at his institution last summer). All submitted papers, including invited papers, have undergone peer review. We hope these articles will present state-of-the-art technological development currently under way and stimulate further novel applications of digital holography and 3-D imaging.

\section{Joint Feature Issue Editors:}

Ting-Chung Poon

Bradley Department of Electrical and Computer

Engineering

Virginia Tech

Blacksburg, Virginia 24061, USA

e-mail: tcpoon@vt.edu

Changhe Zhou

Shanghai Institute of Optics and Fine Mechanics Chinese Academy of Sciences

Qinghe Road 390, Jiading District

Shanghai 201800, China

e-mail: chazhou@mail.shcnc.ac.cn

Toyohiko Yatagai

Utsunomiya University

Center for Optical Research and Education

7-1-2, Yoto, Utsunomiya City, Tochigi 321-8585, Japan

e-mail: yatagai@cc.utsunomiya-u.ac.jp 
Byoungho Lee

School of Electrical Engineering

Seoul National University

Seoul 151-744, South Korea

e-mail: byoungho@snu.ac.kr

\section{Hongchen Zhai}

Institute of Modern Optics

Nankai University, China

Tianjin, 300071, China

e-mail: zhai@nankai.edu.cn 